\title{
Spectrally U-shaped profile of beam propagation factor in all-solid photonic bandgap fiber
}

\author{
Xiao Chen (陈 潇), Liangjin Huang (黄良金)，Yi An (安 毅), Huan Yang (杨 欢), Zhiping Yan (间志平)， \\ Yisha Chen (陈益沙), Xiaoming Xi (奚小明), Zhiyong Pan (潘志勇), and Pu Zhou (周 朴) \\ College of Advanced Interdisciplinary Studies, National University of Defense Technology, Changsha 410073, China \\ *Corresponding author: hlj203@nudt.edu.cn \\ **Corresponding author: zhoupu203@163.com \\ Received August 30, 2021 | Accepted October 19, 2021 | Posted Online November 23, 2021
}

\begin{abstract}
We found the beam quality factor $M^{2}$ of the fundamental mode as a function of wavelength is U-shaped in the working photonic bandgap (PBG) of an all-solid PBG fiber (AS-PBGF) for the first time, to the best of our knowledge, and our simulation results also match well with the phenomenon. The normal band that is near the high-frequency edge of the third PBG integrates the lowest $M^{2}$ and single-mode operation simultaneously, while the other two edge regions suffer from anomalous variation of $M^{2}$ versus wavelength. The general applicability of this finding can be further extended to other PBGs and also other representative structures in the AS-PBGF field.
\end{abstract}

Keywords: all-solid photonic bandgap fiber; beam quality factor; U-shaped curve; photonic bandgap.

DOI: 10.3788/COL202220.010602

\section{Introduction}

Large-mode-area (LMA) fibers working with advanced mechanisms have been established as promising candidates for next-generation high-power fiber lasers, benefitting from their potentials in mode area scaling, higher nonlinear threshold, and feasibilities for some functional applications ${ }^{[1-6]}$. More recently, a kind of bandgap-guided microstructure fiber, called the allsolid photonic bandgap fiber (AS-PBGF), has become an emerging research focus ${ }^{[7-12]}$. Free of air-holes structures, AS-PBGF exhibits excellent practical convenience for the high-power platform $^{[13]}$ and portability for the double-cladding design or polarization-maintaining scheme ${ }^{[11]}$. The all-solid structure ensures the ease of cleaving and splicing, making a compact all-fiber configuration possible. Robust single-mode (SM) operation with high loss ratio between the fundamental mode (FM) and high-order modes (HOMs) is achievable due to the opencladding of AS-PBGF ${ }^{[7]}$. By combining the stack-and-draw method with rare-earth doping technology, the production of active AS-PBGF has gradually become an internal technology mastered by only a few organizations ${ }^{[8,9,11,12,14,15]}$. Over kilowatt-level emission by using double-cladding active AS-PBGF has been successfully demonstrated ${ }^{[16]}$. Besides, the multi-resonant and hetero-structured designs further open a new path for the super LMA AS-PBGF design ${ }^{[8,17,18]}$. With all of the above advantages integrated, the controllable distribution of photonic bandgaps (PBGs) of AS-PBGF additionally provides a novel approach for selective wavelength filtering ${ }^{[13,19]}$.
Therefore, AS-PBGF takes most of the prerequisites for highpower laser applications. Currently, the physics of AS-PBGF optical guidance is a fascinating and ongoing topic of research.

SM operation with near-diffraction-limited output places further requirements on the performances of the LMA fiber. With the development of characterization techniques, a large quantity of researches have been focused on spatiotemporal analysis of the modal characteristics ${ }^{[20-22]}$. According to existing reports, the simultaneous consideration of SM and beam quality usually induces unexpected results ${ }^{[23,24]}$. For example, SM performance and a lower $M^{2}$ in the conventional step-index fiber (SIF) are not always absolutely correlated ${ }^{[23]}$. Even if non-negligible HOMs components appear, it is still possible to predict the beam quality close to the diffraction limit $\left(M^{2} \sim 1\right)$, which conforms to Gaussian energy distribution. As for the AS-PBGF, although the light guiding mechanism that operates near the high-frequency edge has been extensively studied ${ }^{[14]}$, there are few studies concentrating on the transverse mode characteristics at present ${ }^{[25]}$. Previous researches have verified that it is relatively easier to realize the SM operation in the higher frequency band $^{[26]}$, but the beam propagation characteristics within the PBG band have not been systematically studied. Inversely, in most cases, the bending-resistant design requiring AS-PBGF operating far away from the high-frequency edge of the working PBG for this region is more sensitive to bending ${ }^{[17]}$. To fully understand the beam propagation characteristics in AS-PBGF, it is essential to extend the investigation of modal characteristics from the high-frequency edge to the whole PBG. In the early 
report, researchers have employed conventional SIF as the reference to analyze and compare the modal characteristics of airholes PBG-guided fibers ${ }^{[26]}$. However, related research is quite lacking when it comes to AS-PBGF.

In this Letter, by employing an in-house fabricated multiresonant AS-PBGF, the guidance property of AS-PBGF within the entire third PBG is thoroughly investigated and analyzed in detail. Under the premise that the SM behavior is quantified by the spatially and spectrally resolved imaging $\left(S^{2}\right)$ method, the wavelength dependence of the beam quality covering the third PBG is simulated and experimentally measured. The evolution of $M^{2}$ exhibits similar band-spread (U-shaped) distribution to the PBG distribution. Further theoretical calculations also extend the U-shaped tendency from the third PBG to other PBGs. In addition, by comparing with the representative results in the AS-PBGF field, the universality of the findings in this work is demonstrated profoundly.

\section{Experimental Results}

The fiber to be investigated is an in-house fabricated multiresonant AS-PBGF, whose cross-section image is shown in the inset of Fig. 1. In this fiber, multiple-cladding-resonance defect cores are employed to enhance the HOMs leakage ${ }^{[10]}$. It is the same sample as the one utilized in our another work, and more details can be found in Ref. [27]. To achieve broadband operations around the $1 \mu \mathrm{m}$ waveband in the third PBG, the cladding size of the fiber is drawn to $190 \mu \mathrm{m}$. Dimensions of the defect core are measured to be $46 \mu \mathrm{m}$ in the corner-to-corner direction and $39.5 \mu \mathrm{m}$ in the flat-to-flat direction. Limited by the measuring range of the optical spectrum analyzer (OSA), part of the transmission spectrum in the second PBG is not recorded here, as depicted in Fig. 1. It should be noted that the left ordinate of Fig. 1 does not describe the actual transmission losses but describes the relative losses between the measured wavelengths, which is calculated from the supercontinuum minus the output spectrum of the core modes. The third PBG covers broadband

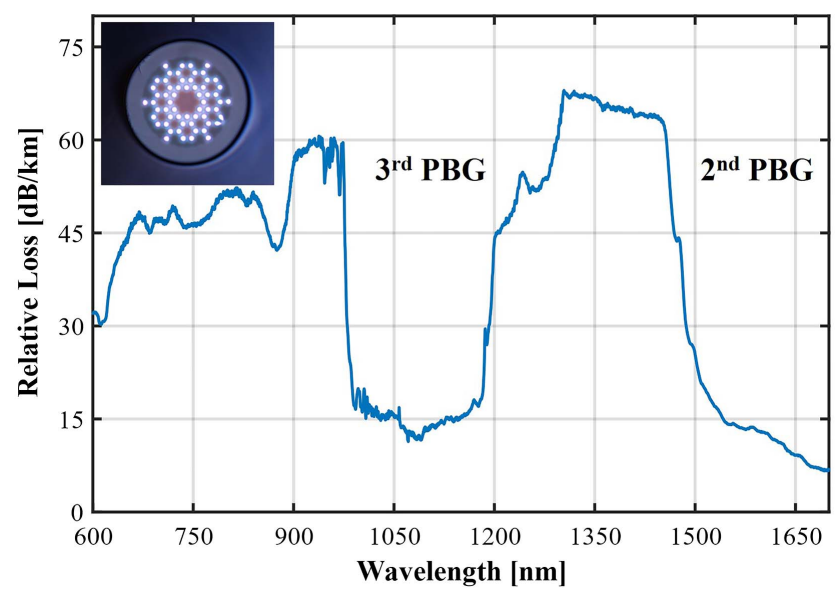

Fig. 1. Relative losses of the 5-m-length fiber. Inset shows the cross section of our multi-resonant fiber. transmission ranging from $990 \mathrm{~nm}$ to $1180 \mathrm{~nm}$, covering the typical operating wavelengths of high-power fiber lasers (e.g., from $1060 \mathrm{~nm}$ to $1090 \mathrm{~nm}$ ). Owing to the bend-insensitive ability and potential for mode area scaling, designs of AS-PBGF in the third PBG are generally preferred in the reported works ${ }^{[11,17,19]}$. Furthermore, limited by the available light sources, the research on the modal characteristics of ASPBGF concentrates on the third PBG in this work. Similar tendency can be further extended and duplicated to the other PBGs, and we will also provide numerical evidences to verify the conclusion theoretically.

The schematics of our experimental setups for $M^{2}$ measurement and SM performance evaluation are depicted in Figs. 2(a) and 2(b), respectively. As illustrated in Fig. 2(a), the tunable source used for $M^{2}$ evaluation integrates a supercontinuum light source (YSL Photonics, SC-OEM) and an acousto-optic tunable filter (AOFT). By adjusting the working frequency of the AOFT, the output wavelength can be artificially controlled to cover the third PBG. Light from the tunable source is launched into the fiber under test by using a butt coupled SM fiber (SMF). To achieve the optimized FM excitation, the SMF is spliced with the AS-PBGF under test directly. In this case, the bending radius of the AS-PBGF is coiled to about $40 \mathrm{~cm}$, so the spectral compression at the high-frequency edge can be avoided to some extent whilst imposing effective HOMs suppression.

The evolution of beam quality factor $M^{2}$ versus wavelength in the third PBG is measured firstly based on the setup shown in Fig. 3(a). As represented in Fig. 3(a), we can see the $M^{2}$ significantly varies with the wavelength in the whole transmission band, especially for the edge regions of the working PBG. The left ordinate scale of Fig. 3(a) has the same definition as the one in Fig. 1. The relative loss curve (black curve) of the third PBG in Fig. 3(a) is a subset of Fig. 1, which represents the third PBG data that is extracted from Fig. 1 specially. Similar to the distribution of PBG curves, the wavelength dependence of $M^{2}$ exhibits the band-spread (U-shaped) feature, but the two curves are not fit entirely. Overall, according to the variation character,

(a)

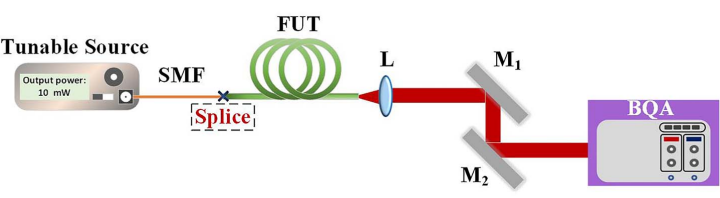

(b)

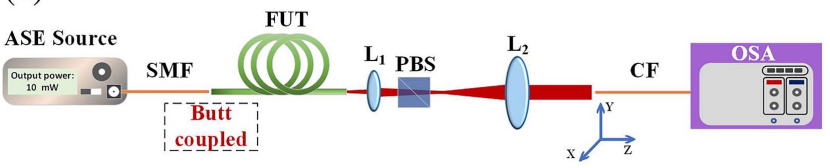

Fig. 2. Experimental configurations constructed for (a) $M^{2}$ and (b) $S^{2}$ measurements, respectively. SMF, single-mode fiber; FUT, AS-PBGF under test; $L_{1} L_{1}$, and $L_{2}$, aspherical lens; $M_{1}$ and $M_{2}$ reflective mirror; $B Q A$, beam quality analysis; ASE source, amplified spontaneous emission source; PBS, polarizing beam splitter; $C F$, collecting fiber; OSA, optical spectrum analyzer. 

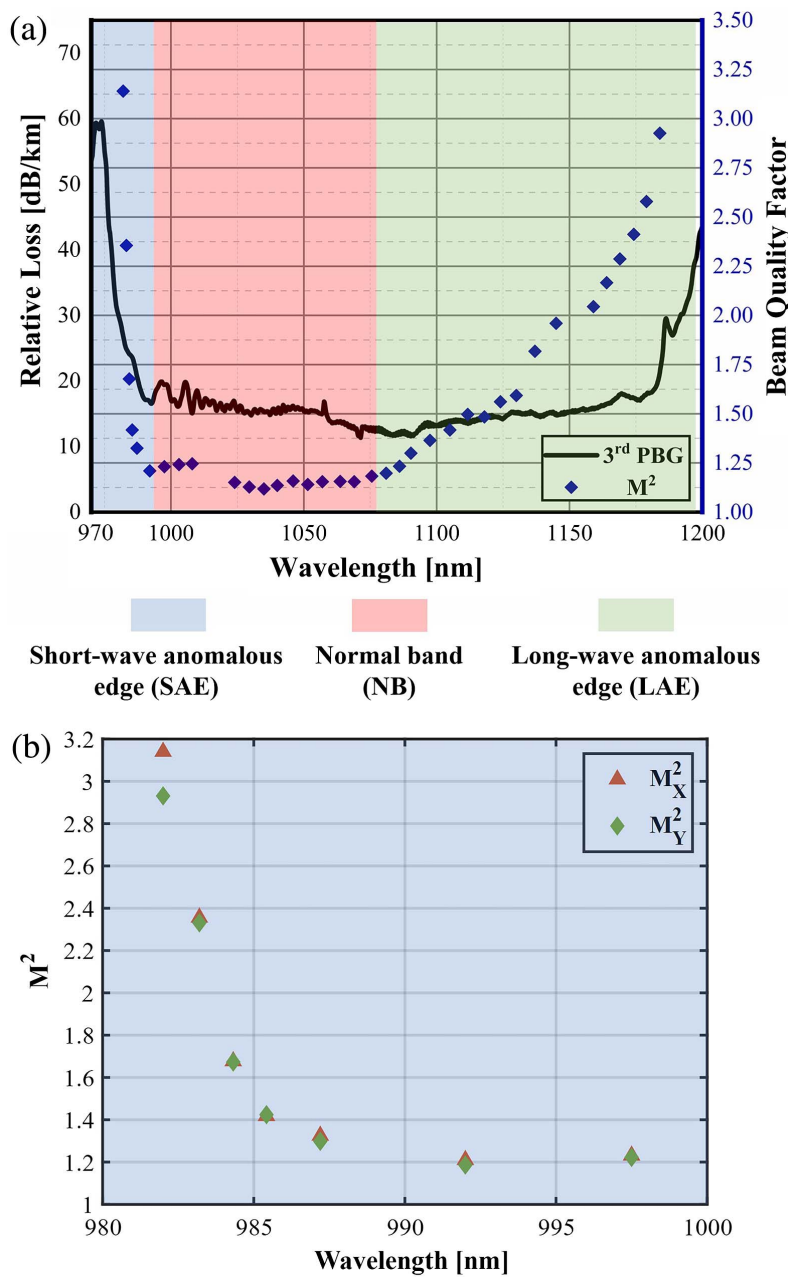

Fig. 3. Measured $M^{2}$ results under different wavelengths. (a) The U-shaped curve covering the third PBG and (b) partial magnification of the results in the SAE region.

the evolution curve of $M^{2}$ in Fig. 3(a) can be divided into three parts, labeled as short-wave anomalous edge (SAE), normal band (NB), and long-wave anomalous edge (LAE), respectively. The above three regions cover $982 \mathrm{~nm}$ to $1000 \mathrm{~nm}, 1000 \mathrm{~nm}$ to $1070 \mathrm{~nm}$, and $1070 \mathrm{~nm}$ to $1185 \mathrm{~nm}$, respectively. At the highfrequency edge of the third PBG, a sharp increment of $M^{2}$ appears with the wavelength shifting to a shorter wavelength in the SAE region. The measured $M^{2}$ rapidly increases to over 2.0 after passing the critical wavelength of near $984 \mathrm{~nm}$, and more details are shown in Fig. 3(b). Within the region of NB, the measured $M^{2}$ keeps relatively stable at the level of less than 1.2 , which is similar to the conventional perception in SIF with SM operation ${ }^{[24]}$. Different from the instantaneous variation of the SAE region, the measured $M^{2}$ increases smoothly as a function of wavelength in the LAE region. The $M^{2}$ value grows gradually with a slope of 0.4 per $50 \mathrm{~nm}$, where the actual value reaches about 1.5 at $1100 \mathrm{~nm}$ and crosses 2.0 at $1150 \mathrm{~nm}$. Within the LAE region, narrow band resonances are enhanced slightly with the variation of wavelength.

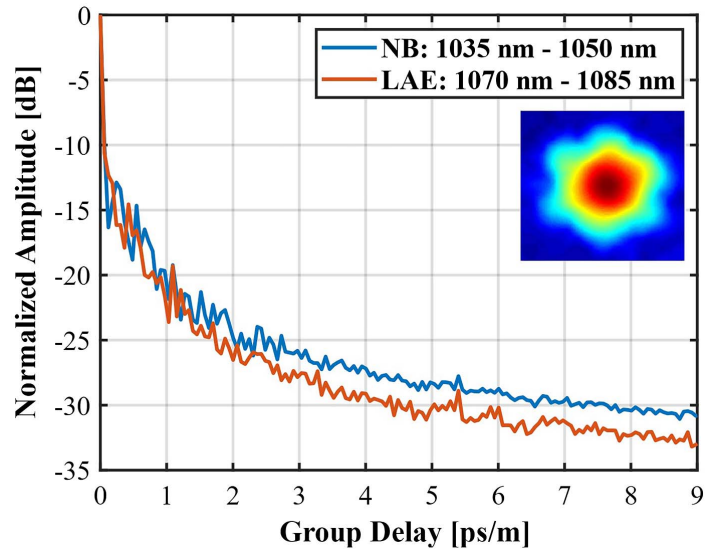

Fig. 4. $S^{2}$ data analysis within the NB and LAE regions with a 5-m-length fiber when the coiled diameter $\Phi=80 \mathrm{~cm}$.

Based on the knowledge of modal characteristics in SIF, the poor $M^{2}$ value normally means considerable contents of HOMs. To monitor the modal contents of the output profile, the $S^{2}$ method is employed to verify the mode spectrum of the output. The setup for performing $S^{2}$ measurements ${ }^{[20,28]}$ employs a broadband emission source, as shown in Fig. 2(b). Light from the source is launched into the AS-PBGF by using a butt coupled SMF, and the fiber output beam is then swept by a cleaved SMF. The collected light is sent to an OSA that captures the interference pattern at each $(x, y)$ point with the wavelength scanning resolution of $0.02 \mathrm{~nm}$.

Figure 4 represents the results of the $S^{2}$ measurements within the different regions of Fig. 3(a). The Fourier transform extracts and averages all spectrum data results in the curve of Fig. 4 . The inset shows the reconstructed beam profile of the fundamental frequency, in which the FM characteristic is well identified. For the first measurement within the NB region, the wavelength sweeping range is between 1035 and $1050 \mathrm{~nm}$. This wavelength window results in a high time differential resolution, which is more than sufficient to allow the characterization of the $5-\mathrm{m}$ length AS-PBGF sample. Similarly, the second measurement in the LAE region selects the wavelength range from $1070 \mathrm{~nm}$ to $1085 \mathrm{~nm}$. On the basis of the Fourier transform results, we found no HOMs peaks, so we can only reconstruct the FM profile, demonstrating the SM behavior of the realized fiber under current test conditions. For the SAE region, the loss is too high to collect the signal with reasonable signal-to-noise ratio, even if we try to utilize the supercontinuum source with higher power than the ASE source. However, according to the leakage guidance mechanism of the HOMs, we can reasonably expect that the FM operation is also valid in the SAE region when the other ranges of the whole working PBG are verified to be operated in the FM.

The U-shaped profile of the beam propagation factor in ASPBGF indicates a potential obstacle in maintaining a lower beam quality factor $M^{2}$ while pursuing the SM operation. According to the $M^{2}$ evolution tendency demonstrated here, robust SM operation with a lower $M^{2}$ value is more convenient to be 
achieved in the NB, which is near the short-wave edge of the third PBG. For the future designs of AS-PBGF, by controlling the PBG bandwidth appropriately, it is possible for us to locate the operating wavelength within the NB region. On the one hand, the beam quality factor of the signal emitting from ASPBGF should be optimized by selecting a suitable wavelength range, even if the SM operation is guaranteed. On the other hand, if the beam quality factor of the light from the ASPBGF is measured to far outweigh one, we should be careful enough to draw a conclusion that there should be many HOM contents if we do not have the mode decomposition results.

\section{Simulation and Discussion}

Furthermore, according to the measured structural parameters, we reconstruct the structure of the fabricated AS-PBGF for simulation. Theoretical distributions of PBGs and beam quality factor $M^{2}$ of the FM are calculated by using the finite element method (FEM) and free-space transfer function ${ }^{[29,30]}$, as shown in Fig. 5. Due to the difference between the actual parameters and the ideal ones, there exists a slight shift between the actual PBGs in Fig. 1 and the theoretical locations in Fig. 5. The dimension measurements variation can also affect the PBGs calculation, because the PBGs distribution shows great sensitivity to the high-index nodes diameter. Nevertheless, the experimental results of $M^{2}$ in Fig. 3(a) and the theoretical curves in Fig. 5 are in great agreement in terms of evolution tendency. Both the calculated $M^{2}$ curves in the third PBG and the fourth PBG display evident band-spread character. The unexpected peaks in the third PBG originate from the weak resonance between the core mode and the node modes. Although the current experimental results are limited to the third PBG, further numerical simulation successfully extends the finding of the U-shaped curve to other PBGs and also provides a universal platform to analyze

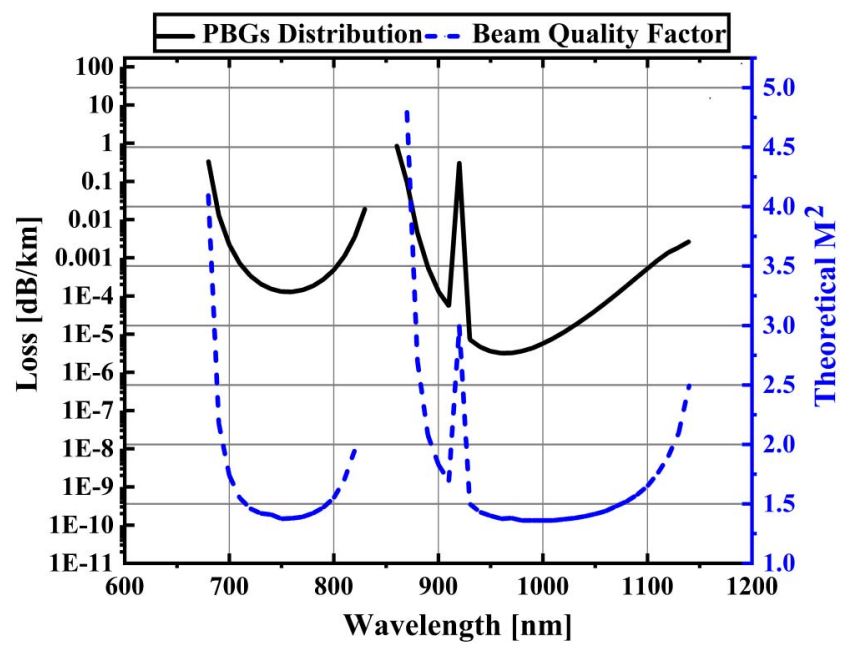

Fig. 5. Theoretical calculations of PBGs distribution and beam quality factor $M^{2}$ based on the measured structural parameters. the wavelength dependence of the beam quality factor in the AS-PBGF.

In view of the unusual modal characteristics of the AS-PBGF compared to conventional SIF, we will further discuss the influence of the FM electric field on the beam propagation property. The FM electric field in the conventional SIF normally maintains a near Gaussian intensity distribution with a flat wavefront, and the value of $M^{2}$ is related to the normalized frequency $V$ to some extent. To be specific, the $M^{2}$ is considered to be close to one when $V$ is greater than $1.5^{[24]}$. Interestingly, based on the measured $M^{2}$ in Fig. 3 and the SM behaviors verified by the $S^{2}$ method within the third PBG, we conclude that even a large $M^{2}$ over two does not mean high HOMs contents, which probably results from the propagation property determined by the light guidance mechanism of the PBG. As for AS-PBGF, all of the guided modes are passively confined in the core region under the tight confinement of the PBG. The multimode cutoff of the microstructural inclusions makes all of the eigenmodes suffer from leakage loss, so there are irremovable side lobes around the eigen $\mathrm{FM}^{[31]}$. The anti-resonance coupling is gradually weakened near the edge of the PBG, and the probability of the core energy passing through the cladding increases. In order to clearly show the difference among the three regions, the beam intensity profiles and phases at three typical wavelengths located in different regions of the third PBG are calculated for comparison, as shown in Fig. 6. Specifically, the $870 \mathrm{~nm}, 1030 \mathrm{~nm}$, and $1140 \mathrm{~nm}$ correspond to the SAE, NB, and LAE regions, respectively. Consistent with the property in conventional SIFs, the central core regions of the three FM distributions show a flat wavefront. The sidelobes result in binary phase with the phase difference reaching $\pi$. Compared with the other two wavelengths, the effective mode area at $870 \mathrm{~nm}$ extends to the cladding region, and the energy of the sidelobes increases significantly. The high-frequency edge (SAE) of the third PBG tends to suffer from higher confinement loss. This phenomenon arises from the difference in the formation of the PBG edges, the effective index mismatch between core modes, and the photonic bands increase that is more rapid at the edge ${ }^{[12]}$. As for $1030 \mathrm{~nm}$ within the NB region, we can see the beam intensity profile is

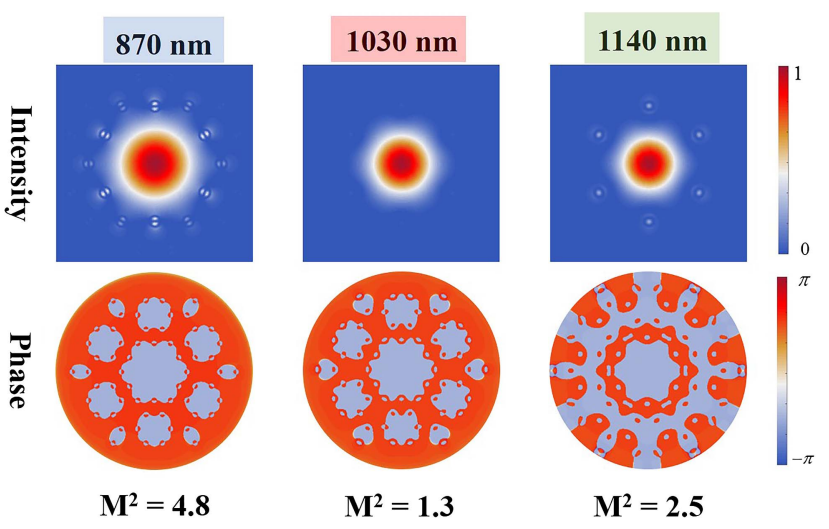

Fig. 6. Theoretical calculations of FM profile and wavefront distribution at $870 \mathrm{~nm}, 1030 \mathrm{~nm}$, and $1140 \mathrm{~nm}$, respectively. 
quite similar to the Gaussian profile with the weakest intensity of sidelobes, which corresponds to a lower $M^{2}$. When it comes to $1140 \mathrm{~nm}$, the main lobe of the intensity profile is similar to the beam at $1030 \mathrm{~nm}$, and there are weak sidelobes around. Different from the cases of $870 \mathrm{~nm}$ and $1030 \mathrm{~nm}$, the binary phase at $1140 \mathrm{~nm}$ appears as a phase ring surrounding the central ground. In this case, even if the core mode is stably confined by the PBG, it will still result in a smooth increment of $M^{2}$.

To extend the universality of the wavelength dependence for $M^{2}$ in the AS-PBGF, two representative results in this field are selected for comparison. The first structure is the "mixed-cell" scheme reported by Gu et al. ${ }^{[18]}$, as depicted in Fig. 7(a), in which the pitch between two adjacent high-index nodes is $\Lambda=25 \mu \mathrm{m}$, the ratio between the diameter $d$ of high-index nodes and $\Lambda$ is $d / \Lambda=0.24$, and the peak value of the high-index profile is $1.48 \%$. Another reference is the "diamond-shaped" scheme proposed by Baz et al. ${ }^{[17]}$, as displayed in Fig. $7(\mathrm{~b})$, where $\Lambda$ is chosen to be $11.8 \mu \mathrm{m}, d / \Lambda=0.79$, and the maximum index contrast of the high-index nodes is $3 \%$. The setting of parameters in the above two structures enables us to locate the operations around $1 \mu \mathrm{m}$ wavelength in the third PBG and fourth PBG, respectively. We numerically calculate the theoretical $M^{2}$ as a function of wavelength in the working PBGs for the above two different design schemes combined with FEM, as illustrated in Fig. $7(\mathrm{c})$. The unexpected peak of the diamond-shaped scheme results from the weak resonance induced by the weak indices matching between the central core mode and the node modes. It is worth emphasizing that these two representative structures also exhibit typical wavelength dependence for $M^{2}$ within the target PBGs. Note that due to the autonomy of simulation model settings, a slight mismatch with respect to the reported results is reasonable, which makes no impact on our conclusion. The existing results show that the wavelength dependence of $M^{2}$ in the AS-PBGF is not an individual case studied in this work but may be a common property that needs further research. In fact, the two schemes also provide representative examples for achieving a lower beam quality factor $M^{2}$ while pursing the SM operation of the AS-PBGF. For instance,
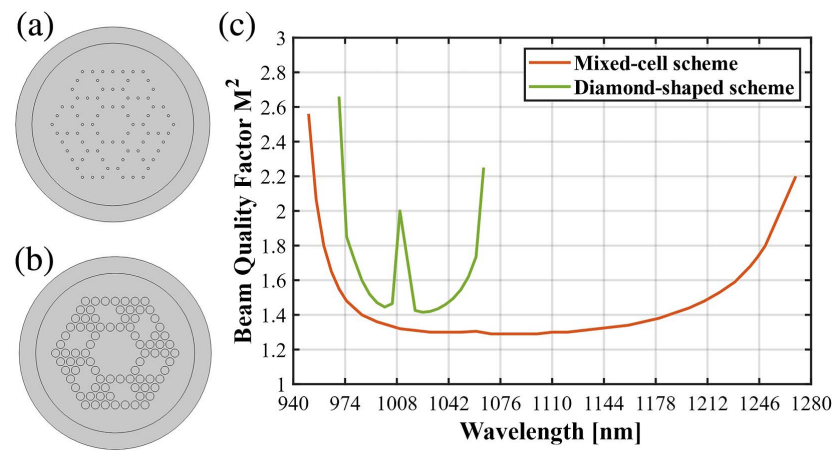

Fig. 7. Beam propagation property of the two representative structures. (a) The "mixed-cell" structure reported in Ref. [18], (b) the "diamond-shaped" scheme proposed in Ref. [17], and (c) calculation results of $M^{2}$ in their respective PBGs. the mixed-cell structure realizes a broadband operation with lower $M^{2}$ values in the third PBG by optimizing the value of $d / \Lambda$, in which the NB region spans from $980 \mathrm{~nm}$ to $1210 \mathrm{~nm}$. The diamond-shaped scheme can be seen as a narrowband example to locate the operating wavelength within the $\mathrm{NB}$ region by compressing the PBG bandwidth appropriately.

\section{Conclusion}

We have theoretically and experimentally verified the spectrally U-shaped profile of the beam propagation factor in the ASPBGF. The measurement result of $M^{2}$ in the third PBG shows a band-spread (U-shaped) feature that is similar to the PBG distribution. In the different regions of the third PBG, the measured $M^{2}$ evolves with different trends. According to the simulation results, the same conclusion is portable when extended to other PBGs. By comparing with two representative structures in this field, the universality of the wavelength dependence of $M^{2}$ in the AS-PBGF is further verified to some extent. In view of the future designs of AS-PBGF or other PBG-guided fibers, this finding seems essential to instruct a scheme for maintaining the beam quality factor while optimizing its SM behavior.

\section{Acknowledgement}

This work was financially supported by the National Natural Science Foundation of China (Nos. 62035015 and 61805280), the Innovation Group of Hunan Province, China (No. 2019JJ10005), the Research Plan of National University of Defense Technology (No. ZK19-07), and the Open Research Fund of State Key Laboratory of Pulsed Power Laser Technology (No. SKL2020ZR07).

\section{References}

1. C. Xie, T. Ning, J. Zheng, L. Pei, J. Wang, J. Li, H. You, C. Wang, and X. Gao, "Amplification characteristics in active tapered segmented cladding fiber with large mode area," High Power Laser Sci. Eng. 9, e32 (2021).

2. X. Zhang, S. F. Gao, Y. Y. Wang, W. Ding, and P. Wang, "Design of large mode area all-solid anti-resonant fiber for high-power lasers," High Power Laser Sci. Eng. 9, e23 (2021).

3. U. Teğin, B. Rahmani, E. Kakkava, D. Psaltis, and C. Moser, "Single-mode output by controlling the spatiotemporal nonlinearities in mode-locked femtosecond multimode fiber lasers," Adv. Photon. 2, 056005 (2020).

4. L. Huang, T. Yao, B. Yang, J. Leng, P. Zhou, Z. Pan, S. Gu, and X. Cheng, "Modified single trench fiber with effective single-mode operation for high-power application," IEEE J. Sel. Top. Quantum Electron. 24, 0901409 (2018).

5. Y. Jeong, J. K. Sahu, D. N. Payne, and J. Nilsson, "Ytterbium-doped large-core fiber laser with $1.36 \mathrm{~kW}$ continuous-wave output power," Opt. Express 12, 6088 (2004).

6. L. Wang, D. He, C. Yu, S. Feng, L. Hu, and D. Chen, "Very large-mode-area, symmetry-reduced, neodymium-doped silicate glass all-solid large-pitch fiber,” IEEE J. Sel. Top. Quantum Electron. 22, 4400105 (2016).

7. L. Dong, F. Kong, G. Gu, T. W. Hawkins, M. Jones, J. Parsons, M. T. Kalichevsky-Dong, K. Saitoh, B. Pulford, and I. Dajani, "Largemode-area all-solid photonic bandgap fibers for the mitigation of optical nonlinearities," IEEE J. Sel. Top. Quantum Electron. 22, 4900207 (2016). 
8. X. Hu, J. Peng, L. Yang, J. Li, H. Li, and N. Dai, “Design and fabrication of a heterostructured cladding solid-core photonic bandgap fiber for construction of Mach-Zehnder interferometer and high sensitive curvature sensor," Opt. Express 26, 7005 (2018).

9. M. Li, L. Wang, S. Han, C. Yu, D. Chen, W. Chen, and L. Hu, "Large-modearea neodymium-doped all-solid double-cladding silicate photonic bandgap fiber with an index step of $\sim 0.5 \%$," Chin. Opt. Lett. 16, 080601 (2018).

10. X. Chen, L. Huang, X. Xi, H. Yang, Y. An, Z. Yan, Z. Pan, and P. Zhou, "Leakage channels enabled multi-resonant all-solid photonic bandgap fiber for effective single-mode propagation," Opt. Express 29, 22455 (2021).

11. O. Vanvincq, A. Cassez, R. Habert, H. E. Hamzaoui, K. Baudelle, S. Plus, D. Labat, M. Bouazaoui, Y. Quiquempois, G. Bouwmans, F. Audo, T. Chartier, E. Lallier, and L. Bigot, "Large mode area solid-core photonic bandgap Yb-doped fiber with hetero-structured cladding for compact high-power laser systems," J. Light. Technol. 39, 4809 (2021).

12. G. Ren, P. Shum, L. Zhang, X. Yu, W. Tong, and J. Luo, "Low-loss all-solid photonic bandgap fiber," Opt. Lett. 32, 1023 (2007).

13. W. Li, T. Matniyaz, S. Gafsi, M. T. Kalichevsky-Dong, T. W. Hawkins, J. Parsons, G. Gu, and L. Dong, "151 W monolithic diffraction-limited Yb-doped photonic bandgap fiber laser at $\sim 978 \mathrm{~nm}$," Opt. Express 27, 24972 (2019).

14. G. Gu, F. Kong, T. Hawkins, J. Parsons, M. Jones, C. Dunn, M. T. Kalichevsky-Dong, K. Saitoh, and L. Dong, "Ytterbium-doped large-mode-area all-solid photonic bandgap fiber lasers," Opt. Express 22, 13962 (2014).

15. M. Li, L. Wang, S. Han, D. Chen, C. Yu, W. Chen, and L. Hu, "Large-modearea neodymium-doped all-solid double-cladding silicate photonic bandgap fiber with a $32 \mu \mathrm{m}$ core diameter," Opt. Mater. Express 8, 1562 (2018).

16. F. Kong, G. Gu, T. Hawkins, M. Jones, J. Parsons, M. Kalichevsky-Dong, B. Pulford, I. Dajani, and L. Dong, " 1 kilowatt ytterbium-doped all-solid photonic bandgap fiber laser,” Proc. SPIE 10083, 1008311 (2017).

17. A. Baz, L. Bigot, G. Bouwmans, and Y. Quiquempois, "Single-mode, large mode area, solid-core photonic bandgap fiber with hetero-structured cladding," J. Lightwave Technol. 31, 830 (2013).

18. G. Gu, F. Kong, T. W. Hawkins, M. Jones, and L. Dong, "Extending mode areas of single-mode all-solid photonic bandgap fibers," Opt. Express 23, 9147 (2015).
19. T. Taru, J. Hou, and J. Knight, "Raman gain suppression in all-solid photonic bandgap fiber," in European Conference and Exhibition of Optical Communication (2007), p. 1.

20. J. W. Nicholson, A. D. Yablon, S. Ramachandran, and S. Ghalmi, "Spatially and spectrally resolved imaging of modal content in large-mode-area fibers," Opt. Express 16, 7233 (2008).

21. D. Flamm, C. Schulze, D. Naidoo, S. Schroter, A. Forbes, and M. Duparre, "All-digital holographic tool for mode excitation and analysis in optical fibers," J. Lightwave Technol. 31, 1023 (2013).

22. Y. An, L. Huang, J. Li, J. Leng, L. Yang, and P. Zhou, "Learning to decompose the modes in few-mode fibers with deep convolutional neural network," Opt. Express 27, 10127 (2019).

23. S. Wielandy, "Implications of higher-order mode content in large mode area fibers with good beam quality," Opt. Express 15, 15402 (2007).

24. H. Yoda, P. Polynkin, and M. Mansuripur, "Beam quality factor of higher order modes in a step-index fiber," J. Lightwave Technol. 24, 1350 (2006).

25. F. Kong, K. Saitoh, D. McClane, T. Hawkins, P. Foy, G. Gu, and L. Dong, "Mode area scaling with all-solid photonic bandgap fibers," Opt. Express 20, 26363 (2012).

26. M. J. F. Digonnet, K. Hyang Kyun, G. S. Kino, and F. Shanhui, "Understanding air-core photonic-bandgap fibers: analogy to conventional fibers," J. Lightwave Technol. 23, 4169 (2005).

27. X. Chen, L. Huang, X. Xi, H. Yang, Y. An, Z. Yan, Z. Pan, and P. Zhou, "Large-mode-area multi-resonant all-solid photonic bandgap fiber with low bending loss and robust single-mode operation," https://ssrn.com/ abstract=3944527 (2021).

28. F. Yu, M. Xu, and J. C. Knight, "Experimental study of low-loss single-mode performance in anti-resonant hollow-core fibers," Opt. Express 24, 12969 (2016).

29. D. Flamm, C. Schulze, R. Brüning, O. A. Schmidt, T. Kaiser, S. Schröter, and M. Duparré, "Fast $M^{2}$ measurement for fiber beams based on modal analysis,” Appl. Opt. 51, 987 (2012).

30. R. Tao, L. Huang, P. Zhou, L. Si, and Z. Liu, "Propagation of high-power fiber laser with high-order-mode content," Photonics Res. 3, 192 (2015).

31. N. M. Litchinitser, S. C. Dunn, B. Usner, B. J. Eggleton, T. P. White, R. C. McPhedran, and C. M. de Sterke, "Resonances in microstructured optical waveguides,” Opt. Express 11, 1243 (2003). 